NCL94-TP12

\title{
Self-similar scalar field collapse: naked singularities and critical behaviour
}

\author{
Patrick R Brady \\ Department of Physics, The University, Newcastle upon Tyne NE1 $7 R U$
}

\begin{abstract}
Homothetic scalar field collapse is considered in this article. By making a suitable choice of variables the equations are reduced to an autonomous system. Then using a combination of numerical and analytic techniques it is shown that there are two classes of solutions. The first consists of solutions with a non-singular origin in which the scalar field collapses and disperses again. There is a singularity at one point of these solutions, however it is not visible to observers at finite radius. The second class of solutions includes both black holes and naked singularities with a critical evolution (which is neither) interpolating between these two extremes. The properties of these solutions are discussed in detail. The paper also contains some speculation about the significance of self-similarity in recent numerical studies.

04.20.Dw, 04.40.Nr, 04.70.Bw
\end{abstract}

Typeset using REVTEX 


\section{INTRODUCTION}

That gravitational collapse leads to black hole formation is widely accepted, yet comparitively little is known about the generic features of collapse. This is well exemplified by the lack of a precise formulation of the cosmic censorship hypothesis [1]. The reason for this is simply our lack of the mathematical tools necessary to analyse the evolution of generic initial data, although some progress is being made on this front [2]. Moreover the complexity of the Einstein field equations counsels retreat to a more tractable system which may capture the essence of the physics. Spherically symmetric general relativity coupled to a massless scalar field is one such system. Indeed this model has been studied in great detail both analytically [3] and numerically [幽,5]. Christodoulou has rigorously established global existence and uniqueness of solutions to the Einstein-scalar field equations and has discussed the general properties of these solutions [3]. He has even established a sufficient condition for the formation of a trapped surface in the future evolution of a given initial data set [6]. Numerical investigations have also provided useful insights into black hole formation. The most recent of these studies, by Choptuik [7], has revealed several intriguing phenomena which were hitherto unknown.

Choptuik considered the numerical evolution of initial data sets characterised by a single parameter ( $p$ say). The resulting families of solutions, $\mathcal{S}[p]$, include both geometries containing black holes, and geometries with only slight deviations from flatness, depending on the value of $p$. Between these two extremes lies a critical evolution with $p=p^{*}$, which signals the transition between complete dispersal and black hole formation. His most interesting results pertain to near critical, $p \simeq p^{*}$, evolutions which exhibit a particularly simple strong field behaviour. In fact, his results strongly suggest that near critical evolutions may be described by a single, universal solution of the field equations. Two quantitative features also emerge from his work: (i) Near critical evolutions contain echoes in the strong field region (i.e. form invariant quantities exhibit a scaling relation like (1.2) below, with $\Delta \simeq-3 \cdot 4)$. Based on his extensive investigations Choptuik has conjectured that the exactly critical solution has 
an infinite train of echoes in the strong field region approaching the singularity. (ii) When black holes form in near critical evolutions the mass scales as $M_{b h} \sim\left|p-p^{*}\right|^{\beta}$, where $\beta$ is an apparently universal constant determined numerically to be about $\cdot 37$

Obtaining analytical results about solutions which exhibit a discrete self-similarity of the type discussed by Choptuik has proved to be extremely difficult [10]. In [11] it has been argued that the piling up of the echoes as the singularity is approached suggests that a continuous self-similarity may be a good approximation in this neighbourhood. Therefore it seems worthwhile to examine collapse under the assumption of a continuous self-similarity. Furthermore I would like to draw attention to a possible explanation of the echoing, discovered by Choptuik, in terms of a continuous self-similarity. In curvature coordinates the spherical line element may be written as

$$
d s^{2}=-\alpha^{2} d t^{2}+a^{2} d r^{2}+r^{2} d \Omega^{2}
$$

where $\alpha$ and $a$ are functions of both $r$ and $t$. Self-similarity implies that there exists a time coordinate such that the metric depends only on the combination $x=r / t$, consequently quantities like $a(r, t)$ satisfy the relation

$$
a\left(e^{\Delta} r, e^{\Delta} t\right)=a(r, t)
$$

for arbitrary values of $\Delta$. Now, consider a coordinate tranformation to a new time, $T$ say, in terms of which $t=f(T)$ where the function $f$ satisfies

$$
f\left(e^{\Delta} T\right)=e^{\Delta} f(T)
$$

only for discrete values of $\Delta$. In terms of this new time coordinate the form invariant quantities examined by Choptuik, along with $a(r / f(T))$, would all appear to have a discrete (rather than continuous) self similarity. How could this be the origin of the discreteness in the numerical work, after all Choptuik [7] states quite clearly that he uses central proper time to describe the solutions - this corresponds to the similarity time $t$ in a spacetime which is everywhere self-similar. However it is likely, and indeed suggested by numerical 
work, that (discrete) self-similarity holds only on intermediate scales, $0<r_{1} \leq r \leq r_{2}$ say. (This has been further highlighted in numerical studies of radiation fluids [9].) Thus it is possible that the time coordinate used in the numerical studies does not correspond to the similarity time. It should be noted that even if this is true the appearance of the universal scaling constant $\Delta \simeq 3 \cdot 4$ continues to be a mystery.

In any case, this paper is concerned with homothetic spacetimes; it is assumed that there exists a vector field $\xi$ such that the spacetime metric satisfies $\mathcal{L}_{\xi} g=2 g$, where $\mathcal{L}_{\xi}$ denotes the Lie derivative with respect to $\xi$. The homothetic collapse of perfect fluids has received a great deal of attention during the past few years [12], where the main thrust of work was searches for naked singularities [13]. The problem of homothetic, scalar field collapse has received comparitively little attention [5], and no examples of naked singularities which evolved from regular initial data were previously known for this form of matter.

The reduction of the scalar field equations to an autonomous system allows me to give a detailed description of all the solutions. Two interesting features emerge from the analysis: Firstly the existence of solutions with naked singularities, and secondly the occurence of phase transitions in the system. The critical point behaviour is of two distinct forms. The first is a phase transition from solutions which (roughly) represent dispersal, to geometries representing black holes. It has been suggested elsewhere 14 that the critical solution implied by the numerical results may be both on the verge of black hole formation and being a naked singularity. We will see below that the second phase transition corresponds to such a situation - the critical evolution lies at the boundary between black holes and naked singularities. In this article the term black hole is used rather loosely to mean that an apparent horizon exists and precedes a central singularity. Of course it is possible to obtain an asymptotically flat solution to the Einstein-scalar equations by cutting off the self-similar evolution at some advanced time and matching it to a less symmetric (not selfsimilar) exterior, in this way one would obtain an asymptotically flat, black-hole spacetime.

The paper is arranged as follows: In section II the field equations for the self-similar collapse are derived in terms of a retarded time coordinate $u$ and a radial coordinate $r$. 
The most general (see appendix A) scalar field evolution consistent with the homothetic symmetry of the spacetime is $\psi=\bar{h}(r /|u|)-\kappa \ln |u|$, where $\kappa$ is an arbitrary (positive) constant. These equations have already been derived in [5] and used to provide initial data in a search for naked singularities. A field redefinition transforms the equations into a nonlinear autonomous system which is amenable to standard techniques [16]. It is then shown that the solutions fall into two distinct classes depending on the value of $\kappa$. Solutions of the first type $(\kappa \geq 1 / \sqrt{4 \pi})$ are shown to be singular only at one point on $r=0$. This singularity is not naked; distant observers cannot see it by waiting a finite proper time. The transition point is at $\kappa=1 / \sqrt{4 \pi}$, although the exactly critical solution actually belongs to the first class.

For each $\kappa<1 / \sqrt{4 \pi}$ there exists a continuous infinity of solutions with a non-singular origin. For a given value of $\kappa$ solutions can represent black holes or naked singularities, with a critical point evolution interpolating between these two extremes. The analytic examples of phase transitions which have been discussed in the literature [11] are of this type, in fact they correspond to the case $\kappa=0$ when the equations can be integrated in closed form. This solution is briefly discussed to explain how it fits into the general analysis presented here. Section IV contains some discussion of the results, and their possible significance.

The notation of [15] is adopted throughout the paper, and detailed calculations which might distract from the main line of thought are relegated to the appendices.

\section{THE FIELD EQUATIONS}

Using retarded Bondi coordinates $\{u, r, \theta, \phi\}$ the spherical line element may be written

$$
d s^{2}=-g \bar{g} d u^{2}-2 g d u d r+r^{2} d \Omega^{2}
$$

where $g=g(u, r), \bar{g}=\bar{g}(u, r)$ and $d \Omega^{2}$ is the standard line element on the unit two-sphere. The origin is singular unless $\bar{g}=g$ when $r=0$. Furthermore it is convenient to normalise the coordinate $u$ so that it represents proper time for an observer at the origin, thus write 


$$
\bar{g}(u, 0)=g(u, 0)=1
$$

The Einstein-scalar field equations are then

$$
\begin{aligned}
(\ln g)_{, r} & =4 \pi r\left(\psi_{, r}\right)^{2}, \\
(r \bar{g})_{, r} & =g, \\
(\bar{g} / g)_{, u} & =8 \pi r g^{-1}\left[\left(\psi_{, u}\right)^{2}-\bar{g} \psi_{, u} \psi_{, r}\right],
\end{aligned}
$$

where a comma denotes partial differentiation. $\psi=\psi(u, r)$ is a massless, minimally coupled scalar field satisfying

$$
\left(\bar{g} r^{2} \psi_{, r}\right)_{, r}=2 r \psi_{, u}+2 r^{2} \psi_{, r u}
$$

Spherical symmetry allows the introduction of a local mass function $m\left(x^{\alpha}\right)$ [19] defined by

$$
1-\frac{2 m\left(x^{\alpha}\right)}{r}:=g^{\alpha \beta} r_{, \alpha} r_{, \beta}=\frac{\bar{g}}{g},
$$

where $r$ is the function which determines the area of the two-spheres. This mass function agrees with both the ADM and Bondi masses in the appropriate limits, and is equivalent to the Hawking quasi-local mass in this case.

\section{A. Self-similar ansatz}

The existence of a homothetic symmetry in a spherical spacetime implies that the metric depend only on $x=r /|u|$, and that the scalar field evolve as

$$
\psi=\bar{h}(x)-\kappa \ln |u|
$$

where $\bar{h}$ is some function to be determined and $\kappa$ is constant (see Appendix A for a proof of this fact). Writing

$$
\bar{h}(x)=\int_{0}^{x} \frac{\gamma(\xi)}{\xi} d \xi
$$


the self-similar equations derived from (2.3)-(2.5) are

$$
\begin{aligned}
(x \bar{g})^{\prime} & =g \\
x g^{\prime} & =4 \pi g(\gamma)^{2}, \\
g-\bar{g} & =4 \pi\left[2 \kappa^{2} x-(\bar{g}-2 x)\left(\gamma^{2}+2 \kappa \gamma\right)\right],
\end{aligned}
$$

where a prime $\left(^{\prime}\right)$ denotes differentiation with respect to $x$. In deriving (2.12) from (2.5) it is necessary to use (2.10) and (2.11) to eliminate derivatives of $g$ and $\bar{g}$. The scalar field evolution is determined by

$$
x(\bar{g}-2 x) \gamma^{\prime}=2 \kappa x-\gamma(g-2 x) .
$$

It is now a straightforward matter to show that (2.10)-(2.12) imply $(2.13)$ provided $\gamma \neq-\kappa$. These equations have been derived previously by Goldwirth and Piran [5] and used to provide boundary conditions in a numerical search for naked singularities.

At the origin (2.2) and (2.12) imply either $\gamma(0)=0$ or $\gamma(0)=-2 \kappa$. Directly evaluating the trace of the stress-energy tensor for the scalar field one finds

$$
T_{\alpha}^{\alpha}=\frac{\bar{g}}{g r^{2}}\left[\gamma^{2}-\frac{x}{\bar{g}}\left(\gamma^{2}+\kappa \gamma\right)\right] \stackrel{r \rightarrow 0}{\longrightarrow} \frac{\gamma^{2}}{r^{2}} .
$$

Clearly the solution can have a non-singular origin only if

$$
\gamma(0)=0
$$

This completes the specification of the initial conditions for the equations $(2.10)-(2.12)$. It is however convenient to recast the system in an autonomous form before discussing the solutions.

\section{B. An equivalent autonomous system}

Analysis of the above equations is facilitated by the field redefinitions

$$
y=\bar{g} / g, \quad z=x / \bar{g},
$$


and the introduction of a new coordinate

$$
\xi=\ln x
$$

Upon substitution into (2.10), (2.11) and (2.13) one obtains the three dimensional, non-linear autonomous system

$$
\begin{gathered}
\dot{z}=z\left(2-y^{-1}\right), \\
\dot{y}=1-\left(4 \pi \gamma^{2}+1\right) y, \\
(1-2 z) \dot{\gamma}=2 \kappa z-\gamma\left(y^{-1}-2 z\right) .
\end{gathered}
$$

The system is effectively two dimensional, however, since $\gamma$ is determined by the algebraic relation

$$
\gamma=-\kappa \pm \sqrt{\frac{\left(1+4 \pi \kappa^{2}\right)-y^{-1}}{4 \pi(1-2 z)}}
$$

provided $y \neq 1 /\left(1+4 \pi \kappa^{2}\right)$. Further discussion is therefore couched in terms of a projection into the $y z$-plane. Consistent with the initial condition (2.15) the positive square root is taken in (2.21), however it must be emphasised that solutions may still evolve continuously onto the other leaf of the surface defined by taking the negative square root above. Indeed it is solutions of this type which have naked singularities.

Requiring that the mass function, defined in (2.7), should be positive or zero implies $y \leq 1$, while $\gamma$ is real only if

$$
\frac{\left(1+4 \pi \kappa^{2}\right)-y^{-1}}{1-2 z} \geq 0
$$

Black hole formation is signalled by $y \rightarrow 0$ (technically this is the condition which locates an apparent horizon in the spacetime). The continuation of the solution to negative values of $y$ will not be considered in the sequel, thus, integral curves of interest lie in the strip $0 \leq y \leq 1$

It should be noted that $\gamma$ is not continuous at

$$
z=1 / 2, \quad y=1 /\left(1+4 \pi \kappa^{2}\right),
$$


thus invalidating the usual existence and uniqueness theorems for systems of ordinary differential equations at this point. This has the important consequence that integral curves of the differential equations may intersect at (2.23). Furthermore, the continuation of such solutions is not always uniquely defined, in some cases there exists an infinite family of possibilities. This is discussed in more detail below and in appendix C.

The character of the solutions shows a strong dependence on the value of $\kappa$. Solving (2.10)-(2.13), subject to the regularity conditions on $r=0$, gives rise to two essentially different classes of solution according as $4 \pi \kappa^{2}$ is greater than or less than unity; they are solutions which do not contain black holes, and solutions which contain either black holes or naked singularities. The critical evolution $\left(4 \pi \kappa^{2}=1\right)$ also belongs to the first class. The transition from one class to the other as one adjusts $\kappa$ is similar to the behaviour discussed by Choptuik [7]. A second type of phase transition from solutions containing black holes to those with naked singularities occurs for each value of $\kappa$ in the range $0<4 \pi \kappa^{2}<1$. This may simply be an artifact of the restriction to self-similar continuations past the point (2.23) however no other continuations are considered here. A similar phenomenon has been observed in Tolman-Bondi collapse [18].

The system (2.18)-(2.20) has two stationary points, one on either leaf of (2.21), given by

$$
y_{ \pm}=\frac{1}{2}, \quad z_{ \pm}=\frac{1}{1 \pm \sqrt{4 \pi} \kappa}, \quad \gamma_{ \pm}= \pm \frac{1}{\sqrt{4 \pi}} .
$$

The nature of these points depends on the value of $\kappa$. It is discussed below and in Appendix B.

\section{SELF-SIMILAR SOLUTIONS}

The self-similar solutions fall quite naturally into two distinct classes depending on the value of $\kappa$. Class I solutions do not contain black holes or naked singularities although they are singular at one point on $r=0$. In the second class are solutions which have naked singularities or black holes; a single critical evolution having a null singularity (which is not 
naked) interpolates between these two extremes. For completeness, at the end of this section it is shown how the results of [11] fit into the overall picture.

\section{A. Class I $-4 \pi \kappa^{2}>1$}

For $\kappa$ in this range only the stationary point $\left(y_{+}, z_{+}, \gamma_{+}\right)$is of interest. This exact solution is equivalently written as

$$
g=2 x(1+\sqrt{4 \pi} \kappa), \bar{g}=x(1+\sqrt{4 \pi} \kappa) .
$$

It has a singular origin $(r=0)$, in fact there are two sheets of this singularity. Future directed, ingoing lightrays terminate on the sheet located at $u=0$, while outgoing lightrays originate on the past sheet (The solution may be obtained by setting $\alpha=\beta=0$ in Eq. (9) of [1]]).

Locally, (3.1) is a positive attractor (see appendix B). The global structure of the Class I solutions is easily determined by examining the behaviour of the integral curves in the region

$$
\mathcal{A}=\left\{1 /\left(1+4 \pi \kappa^{2}\right)<y<1,0<z<1 / 2\right\}
$$

Noting that integral curves enter $\mathcal{A}$ across the lines $y=1 /\left(1+4 \pi \kappa^{2}\right), y=1$ and $\{z=0, y \geq$ $1 / 2\}$ it is evident that the solution originating at $z=0, y=1$ either terminates at the stationary point or leaves $\mathcal{A}$ across $z=1 / 2$. In appendix $\mathrm{C}$ it is shown that integral curves only cross $z=1 / 2$ at $y=1 /\left(1+4 \pi \kappa^{2}\right)$, and that the solution passing through this point is unique when $4 \pi \kappa^{2}>1$. A direct consequence of this is that the solution with a non-singular origin approaches the asymptotic form (3.1), its evolution being characterised by a sequence of decaying oscillations in $y$ about the value $y=1 / 2$. (See Fig. 1)

These solutions do not contain black holes, yet there is a singularity at the origin when $u \rightarrow 0$. Is this singularity naked? Consider ingoing, radial null geodesics

$$
\frac{d r}{d u}=-\bar{g} / 2
$$

Since $x=-r / u$ the geodesics are given by 


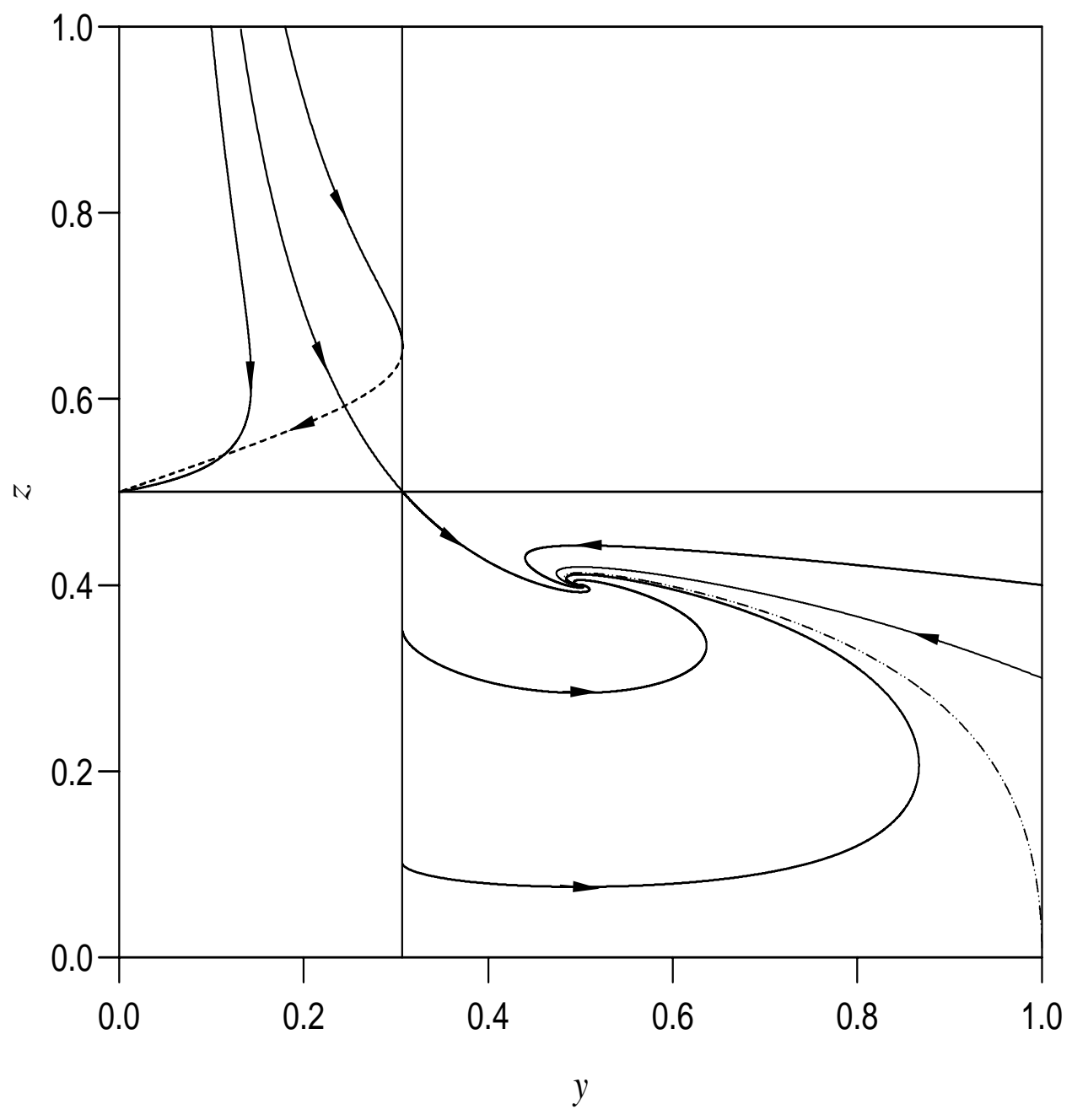

FIG. 1. Integral curves when $4 \pi \kappa^{2}=2.25$. The solutions are seen to spiral towards the stationary solution. The dot-dashed curve from $y=1, z=0$ is the solution with a non-singular origin. The dashed integral curve is on the negative leaf of $\gamma$ and is the continuation of the solid curve which reaches $y=1 /\left(1+4 \pi \kappa^{2}\right)$. 


$$
\ln |u|=2 \int_{x_{1}}^{x}\left(\bar{g}-2 x^{\prime}\right)^{-1} d x^{\prime}
$$

where $x_{1}$ is the radius at the point of intersection between the ingoing geodesic and the null cone $u=-1$. Since $z=x / \bar{g}<1 / 2$ throughout the entire evolution the integrand in (3.4) is always bounded, and $x$ decreases with increasing $u$. Thus ingoing lightrays must reach $r=0$ before $u=0$, and they never reach the singularity provided $x_{1}<\infty$.

In view of the asymptotic solution (3.1) one also finds that an observer at a (large) fixed radius takes an infinite proper time to reach $u=0$. A central observer, on the other hand, reaches the singularity at $u=0$ in finite proper time. It is, however, only after he has seen the entire history of the universe in a tremendous flash. Thus these spacetimes have trivial topology, with a singularity only at $r=0$ as $u \rightarrow 0$.

Similar arguments apply when $4 \pi \kappa^{2}=1$, although in this case $\bar{g}-2 x \rightarrow 0$ as $x \rightarrow \infty$. It must be emphasised that this solution is unstable, in the sense that an arbitrarily small change in the value of $\kappa$ drastically changes the character of the resulting solution. In particular, for smaller values the spacetime contains a black hole.

\section{B. Class II $-4 \pi \kappa^{2}<1$}

Containing two sub-classes of solutions and offering another example of a phase transition in gravitational collapse - from black hole spacetimes to naked singularities - these solutions are more interesting than those in Class I. In fact for each $\kappa$ in the range $0<4 \pi \kappa^{2}<1$ there appears to be a continuous infinity of solutions with a regular origin. This is due to the failure of uniqueness at the singular point (2.23). Naked singularities develop only for sufficiently small values of $\kappa$.

Solutions with a non-singular origin contain a null hypersurface, $\Gamma$ say, on which $x=$ constant. This corresponds to the point $y=1 /\left(1+4 \pi \kappa^{2}\right), z=1 / 2$ in phase space. As mentioned earlier the standard uniqueness theorems break down at this point, and there is a one parameter family of self-similar continuations beyond it. More general extensions which produce asymptotically flat spacetimes have been considered in [5]. 


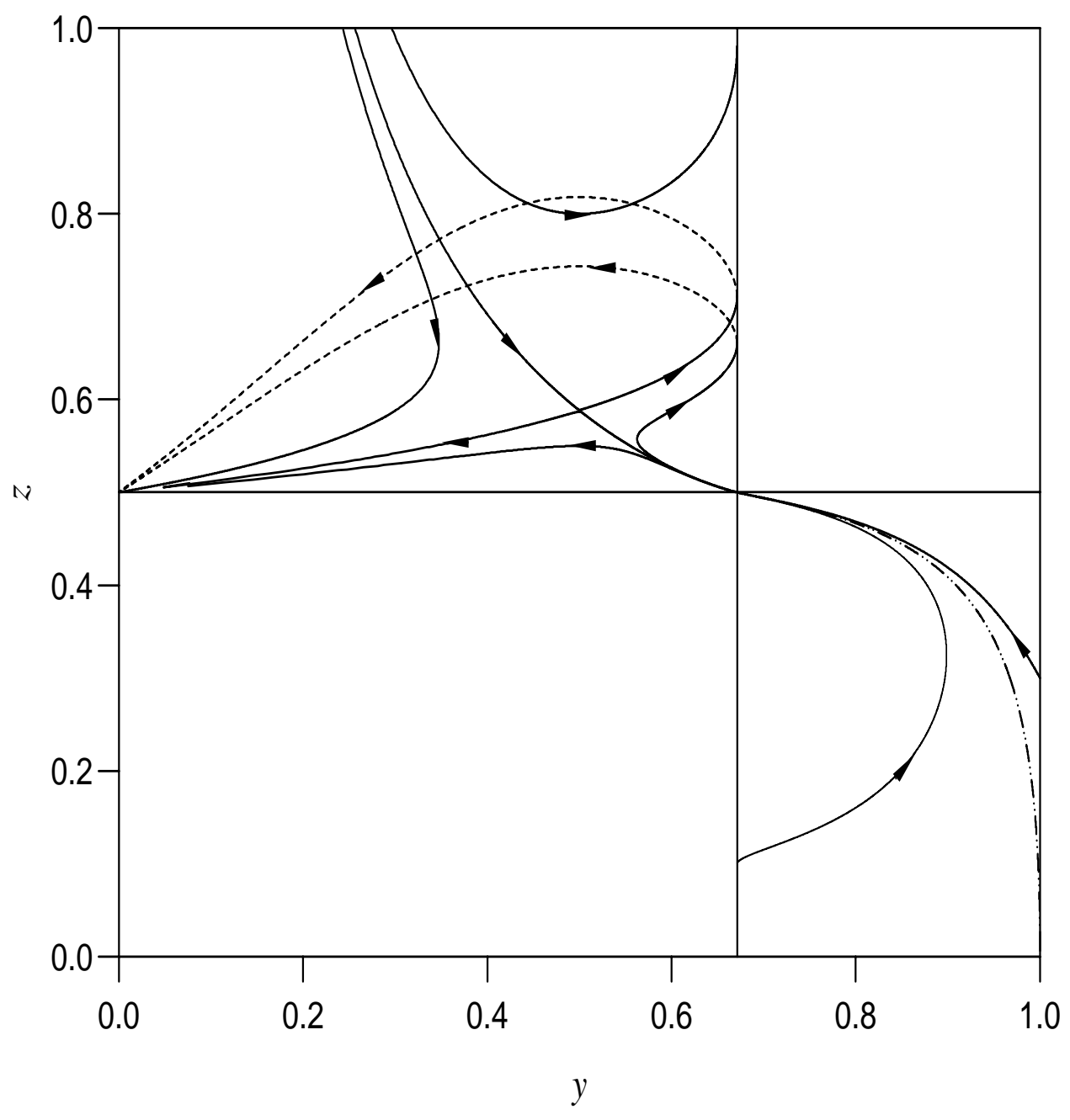

FIG. 2. Integral curves when $4 \pi \kappa^{2}=0 \cdot 49$. Here we see the integral curve representing the solution with a non-singular origin reach the point $z=1 / 2, y=1 /\left(1+4 \pi \kappa^{2}\right)$. There is a one parameter family of continuations past this point. All solutions of interest terminate at $y=0$ (an apparent horizon in spacetime) except one which approaches the stationary point. Here also the dashed lines are integral curves on the negative leaf of $\gamma$. 


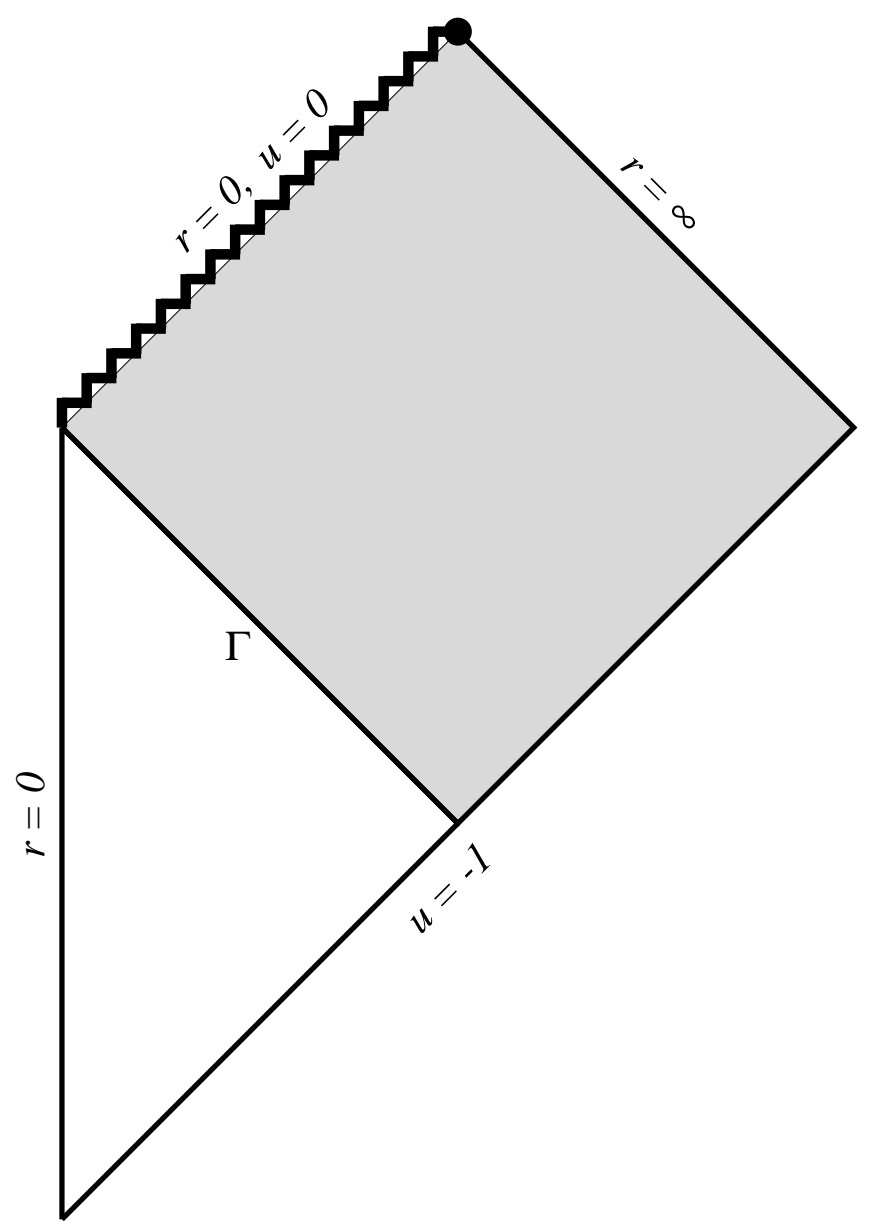

FIG. 3. A spacetime diagram for the critical evolution when $4 \pi \kappa^{2}<1$. The unshaded region corresponds to the dot-dashed curve in Fig. 2 and Fig. 4. The shaded region shows a continuation past $\Gamma\left[\right.$ where $\left.z=1 / 2, y=1 /\left(1+4 \pi \kappa^{2}\right)\right]$ which approaches a stationary point. The singularity at $r=0$ is null. 
Fig. 2 shows various solutions to the system (2.18)-(2.20) when $4 \pi \kappa^{2}=0 \cdot 49$. It is clear from the diagram that all solutions (except one) evolve into black holes (signalled by the formation of an apparent horizon, $y \rightarrow 0$ ). Some of the solutions evolve onto the negative leaf of (2.21); $y$ undergoes a single oscillation before decreasing monotonically to $y=0$. While I have no analytic proof that all of these solutions contain black holes, the numerical results (as illustrated in Fig. 2) suggest this is true. The single exceptional solution exhibits a behaviour which has been discussed elsewhere [11]; beyond $\Gamma$ no black hole forms, instead the solution asymptotically approaches one of the stationary points (2.24). The spacetime is singular at the null surface $u=0, r=0$ where $y=1 / 2$. This singularity lies at infinite redshift for observers at large radius. Fig. 3 is a spacetime diagram for this exceptional case.

For smaller values of $\kappa$ more complicated behaviour is possible, and indeed another type of phase transition is apparent - from black holes to naked singularities. When $z>1 / 2$ the $x=$ constant surfaces are spacelike. If no black hole forms then $x \rightarrow \infty$ as $u \rightarrow 0$ [see Eq. (3.4)], corresponding to a Cauchy horizon in the spacetime. For sufficiently small $\kappa$ there are solutions which evolve from the singular point onto the negative leaf of (2.21), asymptoting to $y=1 /\left(1+4 \pi \kappa^{2}\right)$ as $z \rightarrow \infty$. A typical solution of this type is shown in Fig. 4 , for $4 \pi \kappa^{2}=0 \cdot 25$. It is not difficult to obtain an approximate solution in the large $z$ limit, and hence to show that the Cauchy horizon is non-singular. For large $z$ Eq. (2.19) becomes

$$
\dot{y} \simeq 1-\left(1+4 \pi \kappa^{2}\right) y .
$$

Integrating and inserting the result in (2.18) implies

$$
\begin{aligned}
& y \simeq \frac{1}{1+4 \pi \kappa^{2}}+c x^{-\left(1+4 \pi \kappa^{2}\right)} \\
& z=\frac{x}{\bar{g}} \simeq d x^{1-4 \pi \kappa^{2}}
\end{aligned}
$$

where $c$ and $d$ are arbitrary constants of integration. It is now straightforward to verify the regularity of the Ricci scalar on the Cauchy horizon, using (2.21) and (2.14)

$$
\left.T_{\alpha}^{\alpha}\right|_{u=0}=\frac{\kappa^{2}}{\Delta r^{2}} .
$$




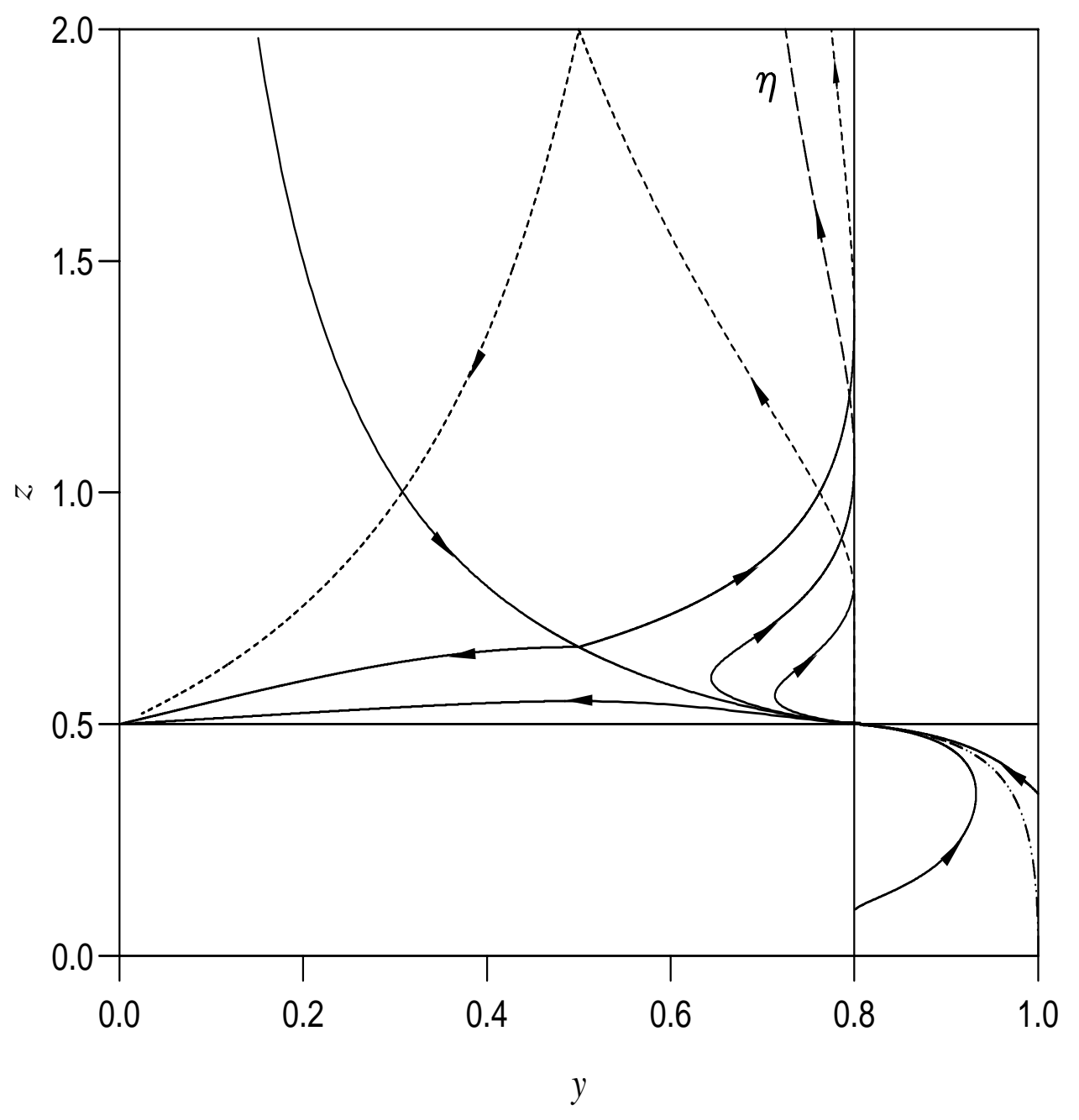

FIG. 4. Integral curves when $4 \pi \kappa^{2}=0 \cdot 25 . \eta$ is an example of an integral curves which evolves onto the negative leaf of $\gamma$ but does not reach $y=0$, instead it attains a minimum value of $y$ and then asymptotes to $y=1 /\left(1+4 \pi \kappa^{2}\right)$. Such solutions have a naked singularity at $r=0$. 
Only as $r \rightarrow 0$ is this quantity singular, indicating the existence of a naked singularity at $u=0, r=0$ in these solutions. The Cauchy horizon is also a null orbit of the homothetic Killing vector, therefore the work of Lake and Zannias [21] implies that the singularity is strong in the sense that tidal forces diverge at it. Notice also that the metric can be made manifestly regular at the Cauchy horizon by transforming to the new coordinate given by

$$
d U=(-u)^{-4 \pi \kappa^{2}} d u
$$

These spacetimes confirm that naked singularities, which evolve from regular initial data, also exist for scalar field sources. Whether they are stable to non-homothethic, not to mention non-spherical, perturbations is an open question although the work of Goldwirth and Piran [5] suggests that they are not.

There are two critical points where phase transitions are observed. Each of the exactly critical solutions, which interpolates between naked singularities and black holes, asymptotically approaches one of the stationary points (2.24). Therefore these spacetimes have the structure shown in Fig. 3, with a null singularity at $u=0, r=0$.

As $\kappa$ decreases further, the topology of the phase space changes slightly; only a single phase transition occurs going from black holes to naked singularities. The solutions (including the single critical evolution) are the same as those already discussed.

\section{The Roberts solution $-\kappa=0$}

When $\kappa=0$ the scalar field is a function of $x=-r / u$ only and the equations (2.10)-(2.12) can be exactly integrated [11]. The resulting solution was first discovered by Roberts [20] and may be written as

$$
\begin{aligned}
& \bar{g}=\left\{\left(p^{2}+x^{2}\right)^{1 / 2}-2 p^{2}\right\} / x \\
& g=2 \bar{g}\left(1 \pm \sqrt{1-4 p^{2}\left(2 x \bar{g}-\bar{g}^{2}\right)}\right)^{-1}
\end{aligned}
$$

and 


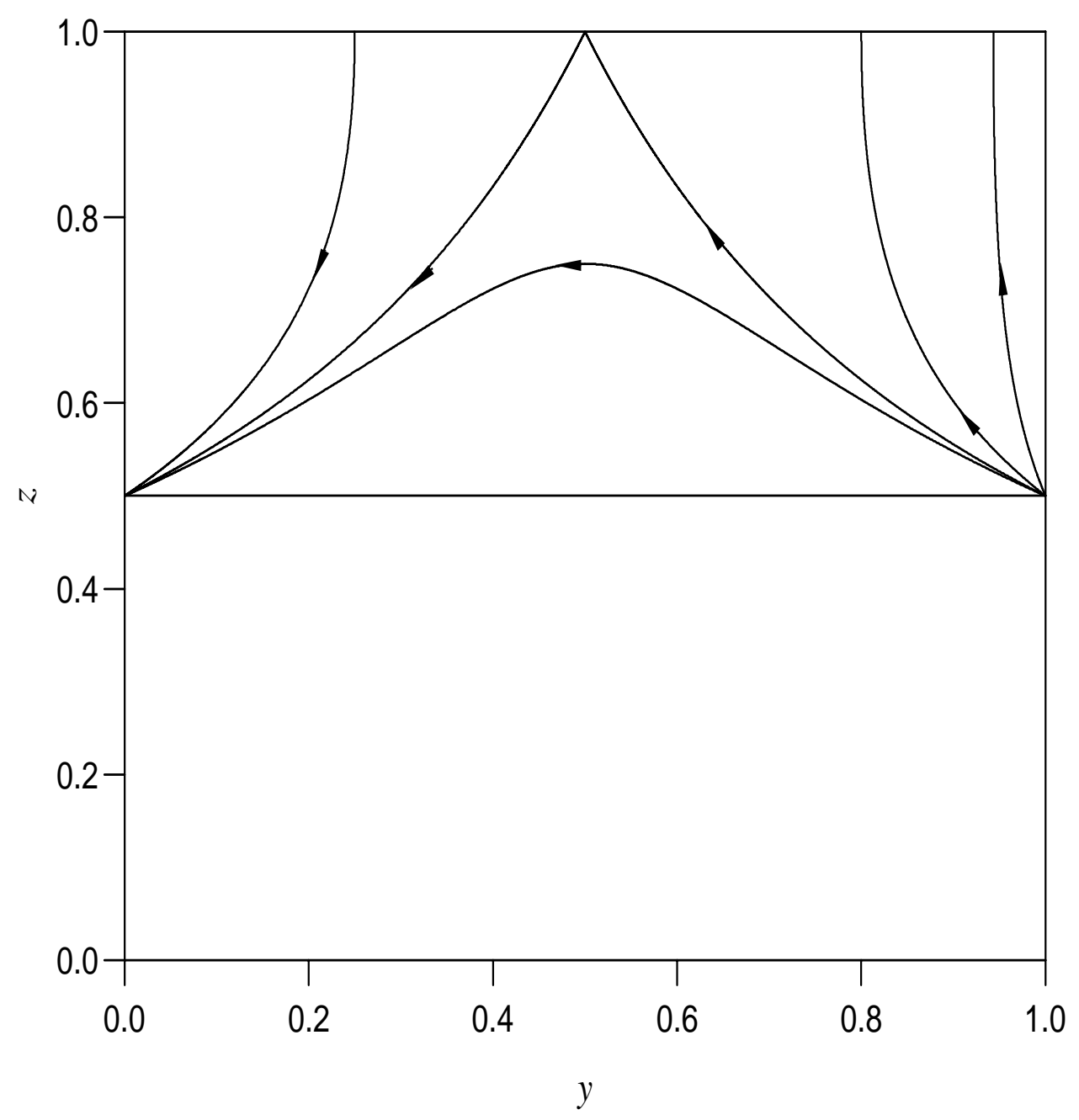

FIG. 5. The exact expression for the solutions when $\kappa=0$ is given in the text, however this diagram is for comparison with the other cases. Note that $\mathrm{y}=1$ is now a solution to the equations and corresponds to flat space. For this reason no naked singularities exist for any value of $p$ when $\kappa=0$. 


$$
\gamma=p / \sqrt{4 \pi\left(p^{2}+x^{2}\right)}
$$

The integral curves are plotted in Fig. 5. The solutions are labelled by $p$ and exhibit critical point behaviour. When $p=1 / 2$ the integral curve leaves $z=1 / 2, y=1$ and approaches the stationary point (2.24). This exactly critical evolution lies between solutions which contain black holes $(y \rightarrow 0, z \rightarrow 1 / 2$ along the integral curves) and those which evolve back to flat space $\left(y \rightarrow 1 /\left(1+4 \pi \kappa^{2}\right), z \rightarrow \infty\right.$ along the integral curves). Since this is a saddle point we see that near critical evolutions (sub- or super-critical) can approach this point arbitrarily closely before moving away in their respective directions.

$\kappa=0$ is therefore exceptional because the subcritical evolutions have zero mass on the Cauchy horizon, $u=0$, as is readily seen by taking the limit $x \rightarrow \infty$ in the above solution. This behaviour is also apparent in the asymptotic approach of the integral curves to the line $y=1$. As discussed in [11 the natural continuation past $u=0$ is Minkowski space since there is no material flux across this surface on which $m=0$. Notice however that there is no self-similar extension of these solutions to $r=0$ which is non-singular.

This discussion places the results obtained in [11] within the more general context of self-similar spacetimes with scalar field matter sources.

\section{DISCUSSION}

Spherically symmetric, homothetic spacetimes have received a great deal of attention over the last few years due to the ease with which it is possible to construct naked singularities in such spacetimes. Recent numerical studies of spherical collapse [7,9] suggest that self-similarity may play an important role in describing the approach to the singularity in gravitational collapse. This study of scalar field collapse was in fact motivated by the work of Choptuik, where he observed discrete self-similarity in solutions on the verge of black hole formation. However it is not clear how a continuous self similarity, as discussed here, could be at the center of the results which he has obtained. Nevertheless some interesting features do emerge from the study of spacetimes with a homothetic symmetry. 
The scalar field evolves as $\psi=\bar{h}(r /|u|)-\kappa \ln |u|$ where $\bar{h}(r /|u|)$ is determined by the coupled Einstein-scalar field equations. The constant $\kappa$ distinguishes between two different classes of solution. The first class (when $4 \pi \kappa^{2} \geq 1$ ) are non-singular almost everywhere. Technically there is a singularity at $u=0=r$, however it is not naked and the worldlines which reach it in finite proper time are a set of measure zero. When $0 \leq 4 \pi \kappa^{2}<1$ the solutions are more interesting - for each value of $\kappa$ in this range there exists infinitely many solutions with a non-singular origin. When $4 \pi \kappa^{2}$ is only slightly less than unity all of these spacetimes contain apparent horizons. However for sufficiently small $\kappa$ some of the spacetimes have naked singularities.

$4 \pi \kappa^{2}=1$ marks the transition point from solutions of Class I to those of Class II - in effect from no black holes to black holes. Another phase transition occurs for each value of $\kappa<1 / \sqrt{4 \pi}-$ a transition from black holes to naked singularities. In particular the critical evolution is on the verge of being a naked singularity in this case. One might wonder if there is some way to classify the critical point behaviour more generally, and to which such class the generic case belongs.

One of the most intriguing results obtained by Choptuik was the scaling law for black hole mass, unfortunately self-similar spacetimes cannot have finite mass black holes. In order to obtain an asymptotically flat spacetime it is necessary to cut off the self-similar evolution at some advanced time, and consider a suitable continuation (which is not self-similar). Goldwirth and Piran [5] did exactly this, although they did not examine the behaviour of black hole mass as the critical point $4 \pi \kappa^{2}$ was approached. This question is currently under active investigation [17], and it will be interesting to see if the mass exhibits the same behaviour which has been observed elsewhere [7,9,8].

We have in self-similar scalar field collapse further examples of spacetimes which violate cosmic censorship. One might be surprised about this were it not for the plethora of examples which now exist. What emerges from these examples (generally) is that there do exist initial data sets which when evolved according to the Einstein equations lead to naked singularities, 
however the genericity of these data is far from clear. It therefore seems that the thrust of any attempt to formulate (and prove) cosmic censorship must address this issue directly. Some interesting preliminary results have been obtained by Lake [22] where he has shown that (spherically symmetric) spacetime in the neighbourhood of a naked singularity may be approximately self-similar. It therefore seems that future work on naked singularities must consider deviations from the symmetric situations treated to date.

Finally in searching for a theoretical understanding of the results obtained by Choptuik [7] and Abrahams and Evans [8] one might consider the obvious generalisation of hometheticity to a conformal symmetry; that is to suppose the existence of a vector field $\xi$ such that

$$
\mathcal{L}_{\xi} g=\Omega(x) g
$$

If the dependence on position in $\Omega$ is weak, solutions might behave like self-similar solutions with some sort of super-imposed periodicity. It would seem interesting to investigate this possibility.

\section{ACKNOWLEDGEMENTS}

I would like to thank Chris Chambers, Peter Hogan, Bruce Jensen, Ian Moss and Adrian Ottewill for useful comments on this work. It is also a pleasure to thank Carsten Gundlach whose comments and questions on an earlier draft of this paper have lead to a significantly different final version. This work was supported by EPSRC of Great Britain.

\section{APPENDIX A: SCALAR FIELD EVOLUTION IN SPHERICAL, SELF-SIMILAR SPACETIMES}

Results of Defrise-Carter [23] imply that a spherical spacetime with a homothetic symmetry (i.e. there exists a vector $\xi$ such that $\mathcal{L}_{\xi} g=2 g$ ) can be written in the form

$$
d s^{2}=e^{2 t}\left(g_{1}(x) d t^{2}+g_{2}(x) d x^{2}+e^{2 x} d \Omega^{2}\right)
$$


where $d \Omega^{2}=d \theta^{2}+\sin ^{2} \theta d \phi^{2}$, and the similarity vector is $\xi=\partial / \partial t$.

The exponential dependence of the metric on $t$ gaurantees that the Christoffel symbols and hence the Ricci tensor, $R_{\mu \nu}$, are independent of this coordinate. This may be expressed covariantly as

$$
\mathcal{L}_{\xi} R_{\mu \nu}=0
$$

Before examining the implication of this for a self-similar spacetime which satisfies Einstein's equations with scalar field matter, let me show that (A1) can be recast into the form used in section II.

Introduce new coordinates $r$ and $u$ defined by

$$
r=\exp (t+x), \quad u=r G(x)
$$

where $G(x)$ is to be determined. Substituting them into the line element (A1) and requiring $u$ to be null one obtains the ordinary differential equation

$$
\frac{d G}{d x}=G\left(-1 \pm \sqrt{-g_{2}(x) / g_{1}(x)}\right)
$$

which determines $G(x)$. Furthermore the line element reduces to

$$
d s^{2}=-\bar{g}(r / u) g(r / u) d u^{2}-2 g(r / u) d u d r+r^{2} d \Omega^{2}
$$

where $r / u$ is realted to $x$ by (A3). Clearly this means that $g$ and $\bar{g}$ are functions of $r / u$ as stated in section II. The similarity vector is

$$
\xi=r \partial_{r}+u \partial_{u}
$$

in these coordinates.

Now in view of (A2) and the Einstein field equations

$$
R_{\mu \nu}=8 \pi \psi_{, \mu} \psi_{, \nu}
$$

the scalar field must satisfy 


$$
\mathcal{L}_{\xi}\left(\psi_{, \mu}\right)=0
$$

Assuming that $\psi$ is independent of $\theta$ and $\phi$, Eq. (A8) is readily integrated to

$$
\begin{aligned}
& \frac{\partial \psi}{\partial r}=\frac{\gamma(r / u)}{r} \\
& \frac{\partial \psi}{\partial u}=\frac{-\gamma(r / u)}{u}
\end{aligned}
$$

where $\gamma$ is an arbitrary function of $r / u$. (Integrability was used to reduce the number of arbitrary functions to one) The general solution of these coupled equations is therefore

$$
\psi=\bar{h}(r / u)-\kappa \ln |u|-\beta \ln |r|
$$

In particular $\beta$ may be set to zero by absorbing $\beta(\ln |u|-\ln |r|)$ into $\bar{h}$, thus reducing (A11) to $(2.8)$.

\section{APPENDIX B: THE STATIONARY POINTS}

The discussion in section III relies on the properties of the stationary points of the equations (2.18)-(2.20). In general there exists two such points given by

$$
y_{ \pm}=\frac{1}{2}, \quad z_{ \pm}=\frac{1}{1 \pm \sqrt{4 \pi} \kappa}, \quad \gamma_{ \pm}= \pm \frac{1}{\sqrt{4 \pi}}
$$

Provided $4 \pi \kappa^{2} \neq 1$ it is straightforward to linearise about each of these points and hence to analyse the topology of the phase space in their neighbourhoods. The eigenvalues are

$$
\lambda_{1,2}=\frac{1-z_{ \pm}}{2 z_{ \pm}-1} \pm \sqrt{\frac{\left(1-z_{ \pm}\right)^{2}+4\left(2 z_{ \pm}-1\right)}{\left(2 z_{ \pm}-1\right)^{2}}},
$$

where $z_{ \pm}$may be chosen independently of the sign of the square root.

When $4 \pi \kappa^{2}>1$ only $z_{+}$is relevant for the discussion in section III. Both eigenvalues are

real and have the same sign, $\lambda_{1,2}<0$, when $1<4 \pi \kappa^{2} \leq 4 / 3$ so that the stationary point is an attractive node. Once $4 \pi \kappa^{2}>4 / 3$ the eigenvalues become complex conjugate, and since

$$
2 z_{+}-1<0, \quad 1-z_{+}>0
$$


they have negative real part. This is a positive attractor, with spiral behaviour.

When $0 \leq 4 \pi \kappa^{2}<1$ both stationary points are of interest. Simply noting that

$$
2 z_{ \pm}-1>0, \quad 1-z_{+}>0, \quad 1-z_{-}<0
$$

the discussion of both is easily combined. Clearly the eigenvalues are real and have opposite signs since

$$
\left|1-z_{ \pm}\right|<\sqrt{\left(1-z_{ \pm}\right)^{2}+4\left(2 z_{ \pm}-1\right)}
$$

Thus they are saddle points.

\section{APPENDIX C: THE SINGULAR LINE $Z=1 / 2$}

In the above analysis it is important that integral curves cannot cross $z=1 / 2$ except at $y=0$ or at $y=1 /\left(1+4 \pi \kappa^{2}\right)$. We now show that this is so, and derive the solution in the neighbourhood of $z=1 / 2, y=1 /\left(1+4 \pi \kappa^{2}\right)$. The analysis is split into two cases:

(i) Suppose an integral curve crosses $z=1 / 2$ at $y_{0} \neq 1 /\left(1+4 \pi \kappa^{2}\right)$. Writing $z=1 / 2+\zeta$ and considering the $\zeta \rightarrow 0$ limit of (2.18), (2.19) and (2.21) it is a straightforward matter to derive

$$
\frac{d y}{d \zeta} \simeq \frac{\left(1+4 \pi \kappa^{2}\right) y^{2}-y}{(2 y-1) \zeta}
$$

Integrating this equation gives

$$
\ln |\zeta| \simeq \text { constant }+\frac{\left(1-4 \pi \kappa^{2}\right)}{\left(1+4 \pi \kappa^{2}\right)} \ln \left|\left(1+4 \pi \kappa^{2}\right) y^{2}-y\right|
$$

Examining this expression shows that $y_{0}=0$ is the only place where integral curves may intersect $z=1 / 2$.

(ii) It is necessary to treat the case when $\left.\zeta\right|_{y=1 /\left(1+4 \pi \kappa^{2}\right)}=0$ separately since the limit $z \rightarrow 1 / 2$ in (2.21) is more delicate. For this purpose we introduce $\zeta$ as above and write 


$$
y=\frac{1}{1+4 \pi \kappa^{2}}+\eta
$$

where $\eta \ll 1 /\left(1+4 \pi \kappa^{2}\right)$, thus

$$
\gamma \simeq-\kappa \pm \frac{1+4 \pi \kappa^{2}}{\sqrt{8 \pi}} \sqrt{\frac{\eta}{-\zeta}}
$$

Substituting this approximate expression for $\gamma$ into (2.19), and using $\zeta$ as the independent variable we arrive at the equation

$$
\frac{d \eta^{1 / 2}}{d \zeta}-\frac{1+4 \pi \kappa^{2}}{2 \zeta\left(1-4 \pi \kappa^{2}\right)} \eta^{1 / 2} \simeq \pm \frac{\kappa \sqrt{8 \pi}}{(-\zeta)^{1 / 2}\left(1-4 \pi \kappa^{2}\right)}
$$

and find

$$
\eta^{1 / 2} \simeq \frac{\sqrt{8 \pi}}{4 \pi \kappa}(-\zeta)^{1 / 2}+c(-\zeta)^{\left(1+4 \pi \kappa^{2}\right) /\left(1-4 \pi \kappa^{2}\right)}
$$

where $c$ is a constant of integration. Now the initial condition is $\eta=0$ when $\zeta=0$ so that there are two distinct possibilities; when $4 \pi \kappa^{2}>1$ the integration constant must vanish, implying that a single integral curve passes through this point. On the other hand if $4 \pi \kappa^{2}<1$ the constant is not fixed by the initial conditions and there is a one parameter family of curves passing through $\eta=0=\zeta$. It is exactly this fact which gives rise to the variety of solutions in Class II. 


\section{REFERENCES}

[1] R. Penrose, in An Einstein Centenary Survey, S.W. Hawking and W. Israel (eds.), Cambridge University Press, Cambridge (1979).

[2] D. Christodoulou and S. Klainerman, The global nonlinear stability of Minkowski space, (Princeton University Press 1993).

[3] D. Christodoulou, Commun. Math. Phys. 105, 337 (1986); 106, 587 (1986); 587 (1986); 109, 591 (1987); 109, 613 (1987).

[4] M.W. Choptuik, in Frontiers in Numerical Relativity, eds. C.R. Evans, L.S. Finn and D.W. Hobill (Cambridge University Press, Cambridge 1989); R. Gómez and J. Winicour, in Frontiers in Numerical Relativity, eds. C.R. Evans, L.S. Finn and D.W. Hobill (Cambridge University Press, Cambridge 1989); R. Gómez, R.A. Isaacson and J. Winicour, J. Comp. Phys. 98, 11 (1992).

[5] A. Goldwirth and T. Piran, Phys. Rev. D36, 3575 (1987).

[6] D. Christodoulou, Commun. Pure Applied Math., Vol. XLIV, 339 (1991).

[7] M.W. Choptuik, Phys. Rev. Letters 70, 9 (1993).

[8] A.M. Abrahams and C.R. Evans, Phys. Rev. Letters 70, 2 (1993).

[9] C.R. Evans and J.S. Coleman, Phys. Rev. Letters 72, 1782 (1994).

[10] V. Husain, E. Martinez and D. Nunez, "Exact solution for scalar field collapse", preprint Alberta-Thy-4-94, gr-qc/9402021 to appear in Phys. Rev. D; J. Traschen, "Discrete self-similarity and critical point behaviour in fluctuations about extremal black holes", preprint UMHEP-405, gr-qc/9403016.

[11] P.R. Brady, Class. Quantum Grav. 11, 1255 (1994); Y. Oshiro, K. Nakamura and A. Tomimatsu, "Critical behaviour of black hole formation in scalar wave collapse", preprint DPNU-94-04 and gr-qc/9402017. 
[12] M.E. Cahill and A.H. Taub, Commun. Math. Phys. 21,1 (1971); D.M. Eardley and L. Smarr, Phys. Rev. D19, 2239 (1979); D. Christodoulou, Commun. Math. Phys. 93, 171 (1984); R.P.A.C. Newman, Class. Quantum Grav. 3, 527 (1986).

[13] A. Ori and T. Piran, Phys. Rev. D42, 1068 (1990); R.N. Henriksen and K. Patel, Gen. Relativ. Grav. 23, 527 (1991); K. Lake and T. Zannias, Phys. Rev. D41, 3866 (1990).

[14] D.M. Eardley, in Texas/PASCOS 92: Relativistic Astrophysics and Particle Cosmology, eds. C.W. Akerlol and M.A. Srednicki (N.Y. Acad. Sci., New York 1993).

[15] C.W. Misner, K.S. Thorne and J.A. Wheeler, Gravitation (Freeman, San Francisco 1973).

[16] F. Verhulst, Nonlinear differential equations and dynamical systems (Springer-Verlag 1990).

[17] P.R. Brady and J. Smith, paper in preparation.

[18] P.S. Joshi and T.P. Singh, "Phase transitions in gravitational collapse of inhomogeneous dust", gr/qc 9405036.

[19] E. Poisson and W. Israel, Phys. Rev D41, 1796 (1990).

[20] M.D. Roberts, Gen. Relativ. Grav. 21, 907 (1989).

[21] K. Lake and T. Zannias, Phys. Rev. D41, 3866 (1990).

[22] K. Lake, Phys. Rev. Letters 68, 3129 (1992).

[23] See D.M. Eardley, Commun. Math. Phys. 37, 287 (1974). 